\title{
DESIGN OF CHINESE SECOND 25-m VLBI ANTENNA
}

\author{
Xu Pei-yuan \\ Shanghai University of Science and Technology \\ Shanghai, People's Republic of China
}

The first Chinese 25-m VLBI antenna has just been completed at the She-Shan Station of the Shanghai Observatory. In accordance with the plan of the Chinese VLBI Network, the second 25-m VLBI antenna will be established at the Urumqi VLBI Station. It is a shaped symmetric dual reflector Cassegrain antenna and will be equipped with nine feeds from $0.3 \mathrm{GHz}$ to $23 \mathrm{GHz}$. The surface accuracy of the main reflector is $0.65 \mathrm{~mm}$, and the $14-\mathrm{m}$ central part has a better surface accuracy $(0.42 \mathrm{~mm})$ to provide the possibility of making VLBI observations at $44 \mathrm{GHz}$. This antenna has a semihomology design, and its subreflector has a mechanism to focus it in three dimensions. Crossed dipole feeds for the 0.32 and $0.61 \mathrm{GHz}$ bands are located in the middle of the subreflector, and the focussing mechanism will retract it to bring the dipoles to the prime focus. For other frequencies, a corrugated horn will be mounted at the Cassegrain focus. To obtain dual frequency operation at $\mathrm{X}$ and $\mathrm{S}$ bands, a flat dichroic reflector and an elliptical reflector will be mounted over the on-axis $\mathrm{S}$ band horn and off-axis $\mathrm{X}$ band horn, respectively. The antenna has a wheel and track mounting, and the pointing will be controlled by an IBM $\mathrm{PC} / \mathrm{XT}$ microcomputer. The pointing and tracking accuracy is 16 arcsecond. The estimated aperture efficiencies are in the range of $45-65 \%$. The estimated antenna temperatures are in the range of 35-100 K. 